\title{
REVIEW ON DEEP LEARNING APPROACH FOR BRAIN TUMOR GLIOMA ANALYSIS
}

\author{
Mrs. Disha Sushant Wankhede, Dr. Selvarani Rangasamy ${ }^{2}$ \\ ${ }^{1}$ Research Scholar, Department of Computer Science and Engineering, Alliance college of engineering, \\ Alliance University, Bangalore, India \\ ${ }^{2}$ Professor-Dept of Computer Science and Engineering, Program director-doctoral studies- ACED, \\ Alliance college of engineering, Alliance University, Bangalore, India \\ disha.wankhede@viit.ac.in
}

\begin{abstract}
Brain tumor diagnosis has evolved as a very critical need in current medical diagnosis. Early diagnosis of tumor detection is an important need for the primitive treatment of brain tumor patient increasing the survival rate of patient. MRI diagnosis of brain tumor for cancer treatment is a large processing due to volumetric content of scan sample. The processing of clinical data is large and consumes a high processing time. Hence, the need of early diagnosis and proper segmentation of brain tumor region is in need. This paper outlines a review on the developments of MRI sample processing for early diagnosis for brain tumor glioma diagnosis using deep learning approach. The advantage of learning capability and finer processing efficiency has gained an advantage in MRI image processing, which enable a better processing efficiency and accuracy in early diagnosis. Deep learning approach has shown a benefit of image coding based on selective features and state of art processing in diagnosis. The evaluation objective of the MRI sample processing has shown a better accuracy than the comparative existing approaches. The recent trends, the advantages and limitation of the existing approach for MRI diagnosis is outlined.
\end{abstract}

Keywords: Deep learning approaches, MRI sample diagnosis, automated processing, Brain tumor glioma analysis.

\section{Introduction}

Caners are developed as a uncontrolled and unnatural development of cells in a part of body region. The development of three cells in brain region is termed as brain tumor. Brain tumor is detected as the most critical among all cancer types [1]. Based on the origin of tumor are defined as primary or metastatic tumor. Wherein primary tumors are developed from the cell tissues, metastatic cells are developed from other body part region and spread into the body region. Gliomas are generated from the glial cells in the brain. The glioma tumor is detected as a major area of tumor detection in brain cancer. Glioma is defined as a general definition of differ types of gliomas which are of low grade such as astrocytomas and oligodendro gliomas and high grade glioblastoma multiform (GBM), which is the most effective malignant primary brain tumor [2]. Chemotherapy, radiotherphy are few methods applied in the treatment of gliomas [3]. An analysis of different medical images such as, Computed Tomography (CT), Positron Emission Tomography (PET), Magnetic Resonance Spectroscopy (MRS), Single-Photon Emission Computed Tomography (SPECT) and Magnetic Resonance Imaging (MRI) are used in the diagnosis of early prediction of cancer in patients. Various 
computer-aided diagnosis (CAD) systems were presented in past for the automated diagnosis of the scan image in early detection of brain tumor. Different optimal algorithms and architectures were proposed in developing an accurate and more efficient classification of brain tumor in glioma diagnosis. In recent development machine learning is observed to evolve as a promising solution in early cancer diagnosis. Among these developments, deep learning approaches were proposed for faster operation with improved accuracy. In building a deep learning approach, the selection of multiple layers in learning and classification system is not optimized. This results in higher computation overhead. The segmentation is limited with edge regions and a predefined reference model is used in making decision. This limits the application of segmentation and hence accuracy of the system. Current diagnosis or computing approach introduces processing distortions which are to be eliminated for accurate processing. The variability of noise characteristics limits the image filters in removing all kinds of noises in variant environments.

Wherein effort in over fitting issue is addressed in diagnosis approach using deep learning approaches, the representative features are very sensitive to distortions introduced during computation. As the features are processed over the whole MRI sample, the resulting coefficient overhead is high and the feature extracted are considerably large. In addition, the classification process based on complete MRI sample results in over fitting issue [4].

The effect of distortion is critical for decision as the outlined coefficients are high in number and the distributive in nature. Even with the complex processing of image coefficients, the non-effective representation of the coefficients lead to heavy representing

coefficients which result in very low accuracy and demand high computation to attain quality of service in medical image processing for automation. To achieve higher decision accuracy, the complexity of representation and classification need to be minimized for an effective processing of medical image sample for real time applications. A set of MRI sample showing high grade of glioma from BRATS dataset is shown in figure 1 .

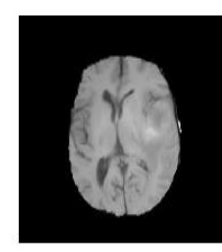

(c)

\section{Figure 1: Different MRI Glioma sample from BRATS dataset}

Figure 1 illustrates the MRI sample where the sub region of tumor region from T1, T1-Gd, T2 and FLAIR is shown in (a),(b),(c),(d) respectively.

For processing of MRI sample developments of new intelligent system in tumor detection were suggested in past. Brain diagnosis and disorder detection for automated tumor detection is presented in [5]. Analysis of the existing cancer detection and the advantages and limitation to the present system is outlined in [6]. The outlined approach defines the feasibility of existing approaches for tumor diagnosis. The feature representation of brain tumor in spatial domain is presented in [7].

A mutual information (MI) is presented for the computation and representation of the spatial feature in [8]. The spatial similar feature for 3-D MRI sample is presented. The process of image feature extraction and registration is outlined. the introduction of distortion in the registration process leading to mis-registration is 
outlined. In [9] the study of brain anatomy and its analysis for studies of tumor detection is presented. The approach gives an extensive learning on the anatomy of brain structure, tumor characteristic and tumor detection approach. In processing of brain tumor detection, segmentation of the region of interest (ROI) is important. In [62] the paper explains IDH1 mutation status and 1P19Q co-deletion status. The process of segmentation used in diagnosis of MRI sample is presented in following section.

\section{Approaches of segmentation in brain tumor diagnosis}

Approach of segmentation is computed by manual segmentation, semi-automated process and fully automated approach [10].

\subsection{Manual Approach}

This approach is based on radiologist refereeing to multiple slices of MRI image and performing a marking of suspected region of tumor in brain. These observations need an expert in anatomical and physiological knowledge of brain image in MRI sample. This process is a time consuming process, and to certain extent of expert based on the variability of sample reflect on the accuracy of detection and are tend to manual error.

\subsection{Semi-Automatic Approach}

This method has a mixed model of manual and automated processing algorithm which are processed for region isolation, processing and feedback analysis for the decision of tumor detection in a MRI sample. This method includes approach such as tumor cut in extracting the tumors region. A suspected manual region is marked onto the MRI sample which is passed to the processing algorithm to make a decision of the marked region.

\subsection{Fully Automatic Region}

This method process isolate on the MRI sample, where advanced computing approaches such as artificial intelligence, machine learning are used in detection of tumor and classification of the detected region.

The past development for segmentation of the tumor region in a MRI sample, a watershed based segmentation approach is defined in [11]. The approach shows the ability of segmenting the required and non-required region in the tumor region segmentation based on the detection of pixel based coding using watershed approach. The method process on the surrounding region coding and the region involved in the process. For segmentation in [12], a graph based technique for region detection is presented. the proposed approach gives an approach of markovian approach to image segmentation using a pair wise coding of region segmentation and registration process.

The approach is developed based on a pattern classification problem, where the registration process is performed using the maximization of similarity index among the region of segmentation.

In order to summarize and analyze the brain tumor's automatic detection methods through the magnetic resonance image (MRI), in [13] various steps of computer-detected detection (CAD) system are presented. Brain image classification was studied in [14] where one way of rating in signal to detect primary brain tumors, combined with multiple wavelets and synthetic neural networks. The EEG signals are measured using unconsciously almost energy. This observation leads to the detection of EEG analysis based tumor. In [15] the images of MRI scan of brain sample is processed on Matlab tool to extract the exact region of the brain tumor. It also includes noise removal functions, side and therapeutic processes that are used for image coding of MRI. To implement and evaluate the method of automated magnetic resonance 
imaging atlas-based automated segmentation (MRI-ABAS) procedure for analyzing patients with brain tumors passing through Radio Therapy (RT) is outlined in [16]. The MRIABAS procedure is developed based on grey matter ratings and atlas -based areas of interest approach. A segmented section of tumorous region is presented in figure 2 below. The segmentation is performed for BRATS glioma sample.
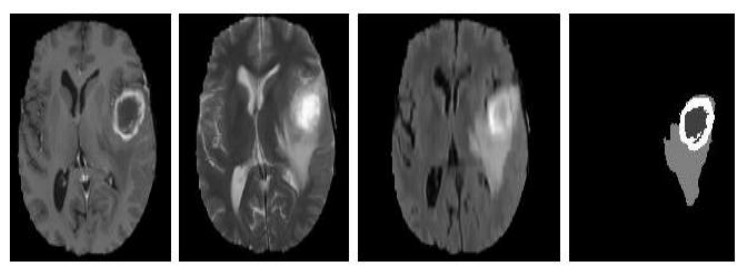

\section{Figure 2: Segmented region of brain tumor from BRATS dataset}

In the segmentation process of a tumorous region in MRI sample, the variation of shape, size and location makes it very challenging task in automated MRI diagnosis. The boundaries of the tumor region are observed to be irregular and the discontinuity of the bounding region poses a greater challenge in segmentation.

\section{Deep Learning Method}

The development in the deep learning methods in various areas of applications such as object recognition, biological image analysis, pattern recognition, speech recognition, security etc., has made the approach more attractive to the research community in recent past [17]. Wherein past learning approach process on feature extraction and selection based on a given user input, deep learning approach such as convolution neural network has a ability of selecting the optimal features from the feature input. Neural network based deep learning approach basically focus on the developing of new network modeling. In decision making of tumor detection in brain tumor detection, deep learning has a important role in accurate region segmentation, feature description and classification.

A 3D convolution neural network based approach for MRI glioma segmentation and recognition is outlined in [18]. This approach presented an approach of handing large spatial feature vectors for neural network modeling and analysis. The proposed approach tested on BRATS dataset gives a dice score of $87 \%$ for the complete tumor region, $77 \%$ for an analysis of core tumor region and $73 \%$ in active tumor region.

[19] Outlined the approach of interpolation of large volumetric data for brain tumor segmentation and feature selection to solve the issue of neural network processing with lower complexity. The proposed method used a hyperbolic tangent function as a non linearity term. This method shows a dice score of $83 \%$ for whole tumor, $73 \%$ for core and $69 \%$ for active tumor region. A two way cascade approach for deep learning in tumor detection is outlined in [20]. This approach implemented a two phase training to avoid class imbalance issue in training process. the Maxout nonlinearity functions used for the post processing. Test of BRATS the dice score obtained are $88 \%$, $79 \%$ and $73 \%$ for the whole, core and active tumor region respectively. A modified rectifier linear unit (ReLU) used a non-linear active function is used in [21]. The neural network uses 11 layers of full connected layers. The obtained dice score are $88 \%, 83 \%$ and $77 \%$ for the whole, core and active tumor region respectively. Local prediction based approach for tumor detection is outlined in [22]. This approach uses a central voxel region for feature extraction and a labeled patch dictionary is used for the development of knowledge database in training process. The obtained dice score for the outlined method is $83 \%, 75 \%$ and $77 \%$ for whole, core and active regions respectively. A summary of the past 
development with dice score tested on BRATS dataset is outlined in table 1 below.
Table 1. Dice Score for Development Deep

Learning approaches for Tumor Glioma Detection on Brats Dataset

\begin{tabular}{|c|c|c|c|c|}
\hline \multirow{2}{*}{ Reference } & Function/Method used & \multicolumn{3}{|c|}{ Dice Score (\%) } \\
\cline { 3 - 5 } & & $\begin{array}{c}\text { Whole } \\
\text { region }\end{array}$ & Core region & $\begin{array}{c}\text { Active } \\
\text { region }\end{array}$ \\
\hline$[18]$ & large spatial feature vectors & 87 & 77 & 73 \\
\hline$[19]$ & hyperbolic tangent function & 83 & 73 & 69 \\
\hline$[20]$ & $\begin{array}{c}\text { two way cascaded approach, Maxout } \\
\text { non-linearity functions }\end{array}$ & 88 & 79 & 73 \\
\hline$[21]$ & modified rectifier linear unit (ReLU) & 88 & 83 & 77 \\
\hline$[22]$ & Local prediction based approach & 83 & 75 & 77 \\
\hline
\end{tabular}

In [23] extensive literature on the approach of developed medical testing for existing methods on tumor detection and its cure is presented. New approach of processing tumor cell detection based on image coding, with classification, detection and recognition is focused. An emphasis on the multimodal, multiscale nature of neuro-imaging data is presented in [24]. The process is developed by a multi-way (tensor) structure, where the defined processes are summarized by a relatively small number of components or "atoms."

[25], focus on the surveillance of tumor detection algorithm that is suggested to detect Tumor's location is presented. The focus is being made on using the image acquisition of brain tumor detection for region of interest segmentation. These approach use MRI images in scanned format to detect tumor in a MRI image. The difference among some of the leading techniques were outlined. In [26] Magnetic resonance imaging using a signalimage post

processing approach is developed using a intensity curvature measurement method. The diagnosis of MRI sample using the curvature process is developed. The brain diagnosis is performed using the functions of the MRI's post processing models namely: (i) bivariate cubic polynomial, (ii) bivariate Lagrange polynomial, (iii) monovariatesinc, and (iv) bivariate linear.

The outcome shows that the conventional approaches of curvature function and signal resilient depends on the signal interpolation and severity to improve the additional information useful for diagnosing for MRI. In [27] DB-4 wavelet is used in extracting the characteristic of MR images. Due to the extraction of finer contrast, it easily reduces the signal variations of an image and reduces the overhead. PCA is applied for the selection of best features for rating. This PCA result is given as input into the SVM selected features for classification. Two SVM kernel functions, linear and radial basis kernels are defined.

These past developments have shown various significant results for automated diagnosis and recognition. However, the learning capability is yet low due to limited feature description, lower filtration efficiency and slower classification process. To overcome these issues, a new intelligent system is outlined. Typical procedures based on special technicians 
are slower, and have low operability and there isolation are to be difficult in quantity. Machine vision seems a suitable technique to automate Computers are used successfully for detection \& classification of brain cancer, segmentation of brain, histogram equalization, thresholding, image enhancement, sharpening filters \& classification of cancers using an artificial neural network approach. A Classification process of deep learning approach on various mode of processing in different dimension of MRI sample is outlined in table 2 .

Table 2. Classification Performance of Tumor Detection for Test Set Sample in Different Dimensions the overall decision of the learning system. Gray level co-occurrence matrix (GLCM) is used in [40] to represent features for a tumor region. In [41] Artificial Bee Colony approach is developed to outlined the feature of tumor using Fuzzy-C-Mine for noise free processing of MRI samples.

In [42] an assessment of current research using data mining techniques for diagnosis of brain tumors is presented. The MRI was diagnosed with the deep learning approach as mining algorithms for extraction of clinical parameters. different methods have been recognized, such as decision trees, support vector machines, synthetic neural networks and multi-peripheral models. The analysis shows

\begin{tabular}{|c|c|c|c|c|c|}
\hline \multirow{2}{*}{ Reference } & \multirow{2}{*}{ Used approach } & \multirow{2}{*}{$\begin{array}{c}\text { Dimension of } \\
\text { coding }\end{array}$} & \multicolumn{3}{|c|}{ Dice score (\%) } \\
\cline { 3 - 6 } & & whole & core & Active \\
\hline$[28]$ & Patch & 3D & 87 & 77 & 73 \\
\hline$[29]$ & Semantic & 2D & 84 & 74 & 69 \\
\hline$[30]$ & cascaded & 3D & 85 & 82 & 81 \\
\hline$[31]$ & cascaded & 2D & 85 & 75 & 57 \\
\hline$[32]$ & Semantic & 2D & 90 & 75 & 77 \\
\hline$[33]$ & Patch & 2D & 89 & 83 & 73 \\
\hline$[34]$ & Semantic & 3D & 90 & 80 & 73 \\
\hline$[35]$ & Patch & 2D & 88 & 84 & 76 \\
\hline$[36]$ & Semantic & 2D & 90 & 88 & 76 \\
\hline$[37]$ & cascaded & 3D & 87 & 78 & 73 \\
\hline$[38]$ & Patch & 2 & & 87 & 72 \\
\hline
\end{tabular}

that most of the data mining algorithm are suitable for brain tumor detection base on the selected feature of training.

In [43] an analysis of transformation techniques, using Discrete Cosine Transformer (DCT), and Discrete wavelet Transform (DWT) is used to rank each other separately as a brain tumor network in integration with probabilistic neural network (PNN) is different. The system was described in three phases for the analysis of tumor in brain. 
In initial phase, MRI images were captured and a pre-processing is carried out to eliminate the noise and to improve the visual quality of the image. secondly, a DCT and DWT approach is compared for feature extraction and a probabilistic neural network based on radial basis function (RBF) is used for the classification of the brain effect. Finally, diagnosis of brain tumor is compared using DCT and DWT and performance parameters using sensitivity rate and precision rate is used. Usage of pre-processing and post processing measures have been presented in [44] for better diagnosis of brain tumor. Six different modes of processing a large scale for measuring area is applied to MRI images. Table 3 outlines the accuracy of estimation based on the method used with the selective features.

Table 3. Classifier Performance with

Feature Vector Used in Tumor Glioma Detection

\begin{tabular}{|c|c|c|c|}
\hline Reference & $\begin{array}{l}\text { Features } \\
\text { used }\end{array}$ & Classifier & $\begin{array}{l}\text { Accuracy } \\
(\boldsymbol{\%})\end{array}$ \\
\hline$[45]$ & Histogram & SVM & 89 \\
\hline$[46]$ & GLCM & SVM & 94 \\
\hline$[47]$ & $\begin{array}{c}\text { Fisher } \\
\text { vectors }\end{array}$ & MPNN & 98 \\
\hline$[48]$ & Fabor & PNN & 83 \\
\hline$[49]$ & GLCM & CNN & 82 \\
\hline$[50]$ & GLCM & CNN & 84 \\
\hline$[51]$ & Selective & CapsNet & 86 \\
\hline
\end{tabular}

\section{System Outline}

The present research work focus on developing robust intelligent system for identifying brain tumor is proposed with reference to the past developed frameworks. The focus on building a new approach for preprocessing, segmentation, feature representation and classification process is addressed. A System architecture for recognition of tumor detection and classification is proposed for identifying brain tumor using intelligent system is as shown in figure 3 below.

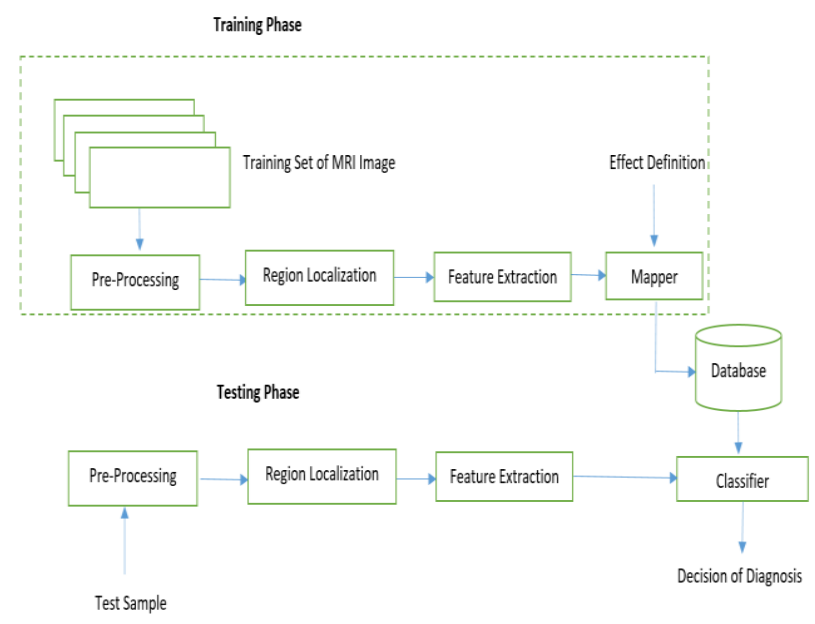

Figure 3: System Architecture for tumor detection

In the process of feature extraction, among different methods Gabor-wavelet approach was mostly used in recent approaches. Whereas Gabor-wavelet approach is more descriptive in texture feature representation, and providing invariance features, the method doesn't reveal much detail of the finer textures at the segmented region. Towards a finer improvement in feature representation a multilevel wavelet with Gabor approach could provide still finer details of the MRI sample. These features can improve the representation accuracy, intern improving the decision accuracy. Such approach will be very effective under detection of tumor at initial stages where the healthy and affected regions are variant at very few locations.

The final stage of this developed approach is the classifier unit, which is dominantly being developed using SVM classifier. SVM classifiers are mostly used due to its simpler processing in implementation and accurate decision. Various formats of kernels were used 
to develop such SVM operation. However, the accuracy of the decision is based on the knowledge it is provided with. In various pattern recognition approaches, a new concept of relevant feedback logic was observed. The relevant feedback logic, improves the accuracy by recursive updation of training knowledge in the SVM model. With this advantage a Multi class SVM (M-SVM) is developed with the integration of relevant feedback logic to improvise the decision accuracy. A summarized decision performance for tumor detection using different methods is listed in table 4 below.

Table 4. Observation of Dice Score for Different Learning Approaches in Tumor Detection for Brats Dataset representation is referred with various common dataset samples. The current dataset consists of 274 multimodality MRI sample in for patient with glioma representing high and low grades effect with ground truth representation for evaluation.

The valuation of the dataset are developed following various known factors of testing metrics such as the Dice-Score, Sensitivity, accuracy, recall rate etc.

Other well known dataset for MRI samples widely used in the diagnosis of MRI samples are,

1) Brain-web data set [63].

2) OASIS [64]

1) Brain-web data set: - currently it has two

\begin{tabular}{|c|l|c|c|c|c|}
\hline Reference & \multicolumn{1}{|c|}{ Used approach } & \multirow{2}{*}{ operation } & \multicolumn{3}{c|}{ Dice score (\%) } \\
\cline { 4 - 6 } & & & whole & Core & Active \\
\hline$[52]$ & Observation and expert entry & Manual & 88 & 93 & 74 \\
\hline$[53]$ & CNN with 3x3 deep learning & Fully automatic & 88 & 83 & 77 \\
\hline$[54]$ & $\begin{array}{l}\text { Joint approach of segmentation and } \\
\text { registration }\end{array}$ & Semi automatic & 87 & 79 & 72 \\
& & & & \\
\hline$[55]$ & Cascade two path & Fully automatic & 87 & 77 & 73 \\
\hline$[56]$ & 3D convolution & Fully automatic & 86 & 77 & 75 \\
\hline$[57]$ & SVM & Semi automatic & 86 & 75 & 73 \\
\hline$[58]$ & CNN k-Mean & Fully automatic & 83 & 74 & 77 \\
\hline$[59]$ & 3D patch CNN & Fully automatic & 85 & 73 & 68 \\
\hline$[60]$ & Cellular automata & Semi automatic & 83 & 73 & 69 \\
\hline$[61]$ & CNN for each modality & Fully automatic & 72 & 57 & 59 \\
\hline
\end{tabular}

(i) Normal

(ii) Multiple sclerosis (MS).

The dataset is represented in two types (T1 and T2 class), where features such as the density, region variant, and intensity of non- linearity features are used in the classification.

2) OASIS (0pen Access Series of Imaging Studies):- The OASIS [64] dataset consist of 150 subject MRI samples of various age groups for tumorous and normal test case. 


\section{Implementation}

We have implemented the system which will identify and analyze whether the given inputted MRI brain scan consists of brain tumor or not shown in Figure 4. Accordingly, it will show the result and display positive or negative along with the accuracy. If the result is found out to be positive, then the segmented portion purpose image will consider.
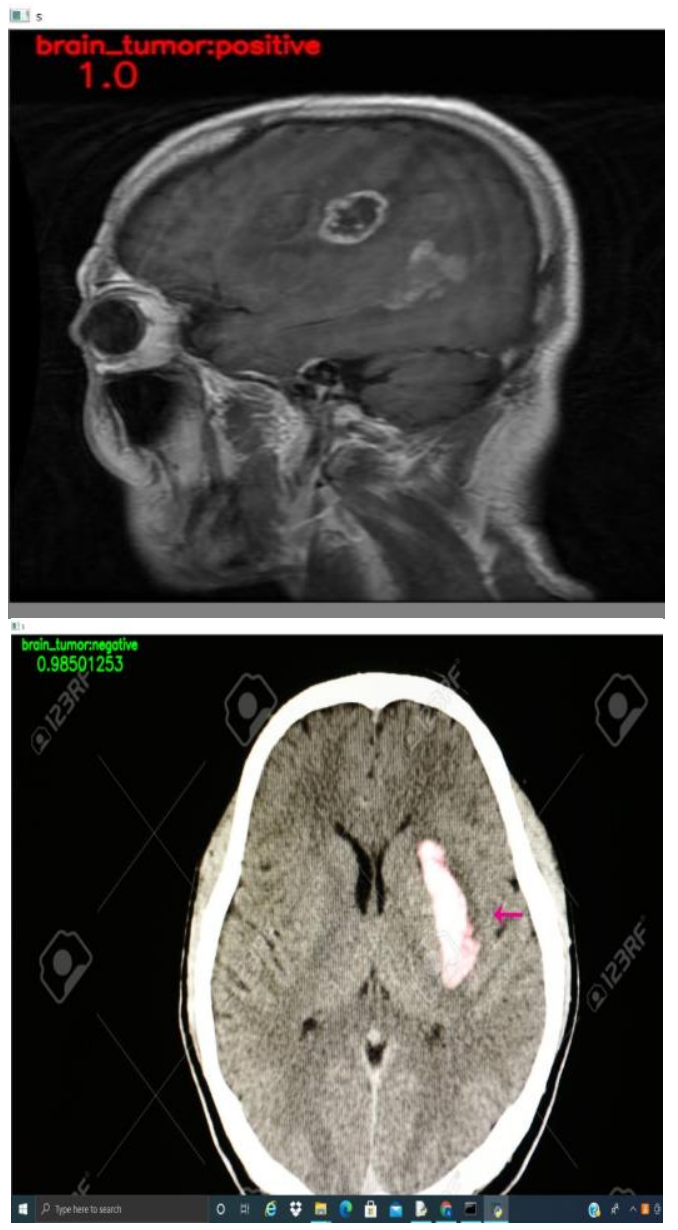

Figure 4: Positive Negative Result

\section{Conclusion}

Automation of brain tumor is observed to be critical due to its volumetric data representation, finer feature details and the processing complexity in making decision. Wherein, accuracy in decision and resource utilization in computation is critical in automation system, processing speed, complexities in computation are also very crucial in these system. Improving the operational performance, recent development has to end towards the incorporation of new machine learning system which has extended to the usage of deep learning approaches. This paper, outlined the developments made in past towards the objective of automation in brain tumor glioma analysis processing with MRI sample. The past approaches of segmentation, feature representation, and classifier models are complex and large in representation. New deep learning approaches such as the neural network $(\mathrm{NN})$ approach offered an advantage of learning and classification using deep processing of tumorous and non-tumorous sample features. The deep learning approach has given an opportunity in developing new approaches in improvising the performance of automation with faster speed and improved accuracy. The implemented model improves the accuracy of the desired results by making a system involving medical image analysis to be carried out by accurate and efficient algorithms. Optimal segmentation, feature selection and classifications are the need of automation system, which are to be defined to the finer level for achieving the objectives of accuracy with speed of operation.

\section{References}

[1] Park, Deric M., and Jeremy N. Rich. "Biology of glioma cancer stem cells." Molecules and cells 28, no. 1 (2009): 7-12.

[2] Van Tellingen, O., B. Yetkin-Arik, M. C. De Gooijer, P. Wesseling, T. Wurdinger, and $\mathrm{H}$. E. De Vries. "Overcoming the blood-brain tumor barrier for effective glioblastoma treatment." Drug Resistance Updates 19 (2015): 1-12.

[3] Davis, Mary Elizabeth. "Glioblastoma: overview of disease and treatment." Clinical journal of oncology nursing 20, no. 5 (2016): S2. 
[4] Hussain, Saddam, Syed Muhammad Anwar, and Muhammad Majid. "Segmentation of glioma tumors in brain using deep convolutional neural network." Neurocomputing 282 (2018): 248-261.

[5] Kaus, Michael R., Simon K. Warfield, Arya Nabavi, Peter M. Black, Ferenc A. Jolesz, and Ron Kikinis. "Automated segmentation of MR images of brain tumors." Radiology 218, no. 2 (2001): 586-591.

[6] Garzon-Muvdi, Tomas, Carmen Kut, Xingde $\mathrm{Li}$, and Kaisorn L. Chaichana. "Intraoperative imaging techniques for glioma surgery." Future Oncology 13, no. 19 (2017): 1731-1745.

[7] Iqbal, Sajid, M. Usman Ghani Khan, Tanzila Saba, and Amjad Rehman. "Computerassisted brain tumor type discrimination using magnetic resonance imaging features." Biomedical Engineering Letters 8, no. 1 (2018): 5-28.

[8] Faranoush, Mohammad, Nami Mohammad Torabi, Azim Mehrvar, Amir Abbas HedayatiAsl, Maryam Tashvighi, Parsa Reza Ravan, Mohammad Ali Fazeli et al. "Classifying pediatric central nervous system tumors through near optimal feature selection and mutual information: A single center cohort." (2013): 153-162.

[9] Swanson, Kristin R., Carly Bridge, J. D. Murray, and Ellsworth C. Alvord Jr. "Virtual and real brain tumors: using mathematical modeling to quantify glioma growth and invasion." Journal of the neurological sciences 216, no. 1 (2003): 110

[10] Hussain, Saddam, Syed Muhammad Anwar, and Muhammad Majid. "Segmentation of glioma tumors in brain using deep convolutional neural network." Neurocomputing 282 (2018): 248-261.

[11] Hasan, SM Kamrul, and Mohiudding Ahmad. "Two-step verification of brain tumor segmentation using watershed- matching algorithm." Brain informatics 5, no. 2 (2018)

[12] Parisot, Sarah, William Wells, Stephane Chemouny, Hugues Duffau, and Nikos Paragios. "Concurrent tumor segmentation and registration with uncertainty-based sparse non-uniform graphs." Medical image analysis 18, no. 4 (2014): 647-659.

[13] Carmona-Fontaine Carlos, Maxime Deforet, Leila Akkari, Craig B. Thompson, Johanna A. Joyce, and Joao B. Xavier. "Metabolic origins of spatial organization in the tumor microenvironment." Proceedings of the National Academy of Sciences 114, no. 11 (2017): 2934-2939.

[14] Sarhan, Ahmad M. "Detection and Classification of Brain Tumor in MRI Images Using Wavelet Transform and Convolutional Neural Network." Journal of Advances in Medicine and Medical Research (2020): 15-26.

[15] Cho, Hwan-ho, Seung-hak Lee, Jonghoon Kim, and Hyunjin Park. "Classification of the glioma grading using radiomics analysis." PeerJ 6 (2018).

[16] Yamanaka, Ryuya, Eisuke Abe, Toshiteru Sato, Azusa Hayano, and Yasuo Takashima. "Secondary intracranial tumors following radiotherapy for pituitary adenomas: A systematic review." Cancers 9, no. 8 (2017).

[17] Wu, Shaocheng, Hongyang Li, Daniel Quang, and Yuanfang Guan. "Three-Planeassembled Deep Learning Segmentation of Gliomas." Radiology: Artificial Intelligence 2, no. 2 (2020).

[18] Rudie, Jeffrey D., David A. Weiss, Rachit Saluja, Andreas M. Rauschecker, Jiancong Wang, Leo Sugrue, Spyridon Bakas, and John B. Colby. "Multi-Disease Segmentation of Gliomas and White Matter Hyperintensities in the BraTS Data Using a 3D Convolutional Neural Network." Frontiers in Computational Neuroscience 13 (2019). 
[19] Scherer, Moritz, Christine Jungk, Michael Götz, Philipp Kickingereder, David Reuss, Martin Bendszus, Klaus Maier-Hein, and Andreas Unterberg. "Early postoperative delineation of residual tumor after low-grade glioma resection by probabilistic quantification of diffusion-weighted imaging." Journal of neurosurgery 130, no. 6 (2018): 2016-2024.

[20] Lachinov, Dmitry, Evgeny Vasiliev, and Vadim Turlapov. "Glioma segmentation with cascaded UNet." In International MICCAI Brainlesion Workshop, pp. 189198. Springer, Cham, 2018.

[21] Khawaldeh, Saed, Usama Pervaiz, Azhar Rafiq, and Rami S. Alkhawaldeh. "Noninvasive grading of glioma tumor using magnetic resonance imaging with convolutional neural networks." Applied Sciences 8, no. 1 (2018).

[22] Liu, Luyan, Han Zhang, Jinsong Wu, Zhengda Yu, Xiaobo Chen, Islem Rekik, Qian Wang, Junfeng Lu, and Dinggang Shen. "Overall survival time prediction for high-grade glioma patients based on largescale brain functional networks." Brain imaging and behavior 13, no. 5 (2019): 1333-1351.

[23] Ghosh, Debarati, Saikat Nandi, and Sonali Bhattacharjee. "Combination therapy to checkmate Glioblastoma: clinical challenges and advances." Clinical and translational medicine 7, no. 1 (2018).

[24] Verburg, Niels, Petra JW Pouwels, Ronald Boellaard, Frederik Barkhof, Otto S. Hoekstra, Jaap C. Reijneveld, W. Peter Vandertop, Pieter Wesseling, and Philip C. de Witt Hamer. "Accurate Delineation of Glioma Infiltration by Advanced PET/MR Neuro-Imaging (FRONTIER Study) A Diagnostic Study Protocol." Neurosurgery 79, no. 4 (2016): 535-540.

[25] Panditharatna, Eshini, Lindsay B. Kilburn, Mariam S. Aboian, Madhuri Kambhampati,
Heather Gordish-Dressman, Suresh N. Magge, Nalin Gupta et al. "Clinically relevant and minimally invasive tumor surveillance of pediatric diffuse midline gliomas using patient-derived liquid biopsy." Clinical Cancer Research 24, no. 23 (2018): 5850-5859.

[26] Wu, Sheng-Kai, Marc A. Santos, Stuart L. Marcus, and Kullervo Hynynen. "MRguided Focused Ultrasound Facilitates Sonodynamic Therapy with 5Aminolevulinic Acid in a Rat Glioma Model." Scientific reports 9, no. 1 (2019): 110.

[27] Panda, Bichitra, and Chandra Sekhar Panda. "A Review on Brain Tumor Classification Methodologies." (2019).

[28] Mlynarski, Pawel, Hervé Delingette, Antonio Criminisi, and Nicholas Ayache. "3D convolutional neural networks for tumor segmentation using long-range 2D context." Computerized Medical Imaging and Graphics 73 (2019): 60-72.

[29] Rezaei, Mina, Konstantin Harmuth, Willi Gierke, Thomas Kellermeier, Martin Fischer, Haojin Yang, and Christoph Meinel. "A conditional adversarial network for semantic segmentation of brain tumor." In International MICCAI Brainlesion Workshop, pp. 241-252. Springer, Cham, 2017.

[30] Wang, Guotai, Wenqi Li, Sébastien Ourselin, and Tom Vercauteren. "Automatic brain tumor segmentation using cascaded anisotropic convolutional neural networks." In International MICCAI brainlesion workshop, pp. 178-190. Springer, Cham, 2017.

[31] Hua, Rui, Quan Huo, Yaozong Gao, He Sui, Bing Zhang, Yu Sun, Zhanhao Mo, and Feng Shi. "Segmenting Brain Tumor Using Cascaded V-Nets in Multimodal MR Images." Frontiers in Computational Neuroscience 14 (2020). 
[32] Sobhaninia, Zahra, Safiyeh Rezaei, Nader Karimi, Ali Emami, and Shadrokh Samavi. "Brain Tumor Segmentation by Cascaded Deep Neural Networks Using Multiple Image Scales." arXiv preprint arXiv:2002.01975 (2020).

[33] Mlynarski, Pawel, Hervé Delingette, Antonio Criminisi, and Nicholas Ayache. "3D convolutional neural networks for tumor segmentation using long-range 2D context." Computerized Medical Imaging and Graphics 73 (2019): 60-72.

[34] Yogananda, Chandan Ganesh, Bhavya R. Shah, Maryam Vejdani-Jahromi, Sahil S. Nalawade, Gowtham K. Murugesan, Frank F. Yu, Marco C. Pinho et al. "A novel fully automated MRI-based deep-learning method for classification of IDH mutation status in brain gliomas." Neuro-oncology 22, no. 3 (2020): 402-411.

[35] Agravat, Rupal, and Mehul S. Raval. "3D Semantic Segmentation of Brain Tumor for Overall Survival Prediction." arXiv preprint arXiv:2008.11576 (2020).

[36] Bakas, Spyridon, Mauricio Reyes, Andras Jakab, Stefan Bauer, Markus Rempfler, Alessandro Crimi, Russell Takeshi Shinohara et al. "Identifying the best machine learning algorithms for brain tumor segmentation, progression assessment, and overall survival prediction in the BRATS challenge." arXiv preprint arXiv:1811.02629 (2018).

[37] Hu, Xiaobin, Hongwei Li, Yu Zhao, Chao Dong, Bjoern H. Menze, and Marie Piraud. "Hierarchical multi-class segmentation of glioma images using networks with multilevel activation function." In International MICCAI Brainlesion Workshop, pp. 116127. Springer, Cham, 2018.

[38] Stawiaski, Jean. "A Multiscale Patch Based Convolutional Network for Brain Tumor Segmentation." arXiv preprint arXiv:1710.02316 (2017).
[39] Belghese, Jibi, and Sheeja Agustin. "Brain Tumor Segmentation using Pattern Neural Networks with MRI Images." IJSTEInternational Journal of Science Technology \& Engineering 3 (2017).

[40] Kunimatsu, Akira, Natsuko Kunimatsu, Kouhei Kamiya, Takeyuki Watadani, Harushi Mori, and Osamu Abe. "Comparison between glioblastoma and primary central nervous system lymphoma using MR image-based texture analysis." Magnetic Resonance in Medical Sciences 17, no. 1 (2018).

[41] Kharis, S. A. A., I. Hadi, and K. A. Hasanah. "Multiclass Classification of Brain Cancer with Multiple Multiclass Artificial Bee Colony Feature Selection and Support Vector Machine." In Journal of Physics: Conference Series, vol. 1417, no. 1, p. 012015. IOP Publishing, 2019.

[42] Zhan, Tianming, Fangqing Shen, Xunning Hong, Xihu Wang, Yunjie Chen, Zhenyu Lu, and Guowei Yang. "A glioma segmentation method using cotraining and superpixel-based spatial and clinical constraints." IEEE Access 6 (2018): 5711357122.

[43] Polly, F. P., S. K. Shil, M. A. Hossain, A. Ayman, and Y. M. Jang. "Detection and classification of HGG and LGG brain tumor using machine learning." In 2018 International Conference on Information Networking (ICOIN), pp. 813-817. IEEE, 2018.

[44] KV, Ahammed Muneer, and V. R. Rajendran. "Glioma tumor grade identification using artificial intelligent techniques." Journal of medical systems 43, no. 5 (2019).

[45] Rathore, Saima, Hamed Akbari, Martin Rozycki, Kalil G. Abdullah, MacLean P. Nasrallah, Zev A. Binder, Ramana V. Davuluri et al. "Radiomic MRI signature reveals three distinct subtypes of 
glioblastoma with different clinical and molecular characteristics, offering prognostic value beyond IDH1." Scientific reports 8, no. 1 (2018): 1-12.

[46] Bahadure, Nilesh Bhaskarrao, Arun Kumar Ray, and Har Pal Thethi. "Image analysis for MRI based brain tumor detection and feature extraction using biologically inspired BWT and SVM." International journal of biomedical imaging 2017 .

[47] Rehman, Arshia, Saeeda Naz, Muhammad Imran Razzak, Faiza Akram, and Muhammad Imran. "A deep learning-based framework for automatic brain tumors classification using transfer learning." Circuits, Systems, and Signal Processing 39, no. 2 (2020): 757-775.

[48] Schiffer, Davide, Laura Annovazzi, Cristina Casalone, Cristiano Corona, and Marta Mellai. "Glioblastoma: microenvironment and niche concept." Cancers 11, no. 1 (2019).

[49] Alqudah, Ali Mohammad, Hiam Alquraan, Isam Abu Qasmieh, Amin Alqudah, and Wafaa Al-Sharu. "Brain Tumor Classification Using Deep Learning Technique--A Comparison between Cropped, Uncropped, and Segmented Lesion Images with Different Sizes." 2001.08844 (2020).

[50] Sajjad, Muhammad, Salman Khan, Khan Muhammad, Wanqing $\mathrm{Wu}$, Amin Ullah, and Sung Wook Baik. "Multi-grade brain tumor classification using deep CNN with extensive data augmentation." Journal of computational science 30 (2019): 174-182.

[51] Afshar, Parnian, Arash Mohammadi, and Konstantinos N. Plataniotis. "Brain tumor type classification via capsule networks." In 2018 25th IEEE International Conference on Image Processing (ICIP), pp. 3129-3133. IEEE, 2018.

[52] Bakas, Spyridon, Hamed Akbari, Aristeidis Sotiras, Michel Bilello, Martin Rozycki,
Justin S. Kirby, John B. Freymann, Keyvan Farahani, and Christos Davatzikos. "Advancing the cancer genome atlas glioma MRI collections with expert segmentation labels and radiomic features." Scientific data 4 (2017).

[53] Tjahyaningtijas, Hapsari Peni Agustin, Andi Kurniawan Nugroho, Cucun Very Angkoso, I. Ketut Edy Purnama, and Mauridhi Hery Purnomo. "Automatic Segmentation on Glioblastoma Brain Tumor Magnetic Resonance Imaging Using Modified U-Net." EMITTER International Journal of Engineering Technology 8, no. 1 (2020): 161-177.

[54] Wu, Yaping, Zhe Zhao, Weiguo Wu, Yusong Lin, and Meiyun Wang. "Automatic glioma segmentation based on adaptive superpixel." BMC Medical Imaging 19, no. 1 (2019): 1-14.

[55] Amiri, Samya, Mohamed Ali Mahjoub, and Islem Rekik. "Tree-based ensemble classifier learning for automatic brain glioma segmentation." Neurocomputing 313 (2018): 135-142.

[56] Decuyper, Milan, and Roel Van Holen. "Fully automatic binary glioma grading based on pre-therapy MRI using 3D convolutional neural networks." arXiv preprint arXiv:1908.01506 (2019).

[57] Wu, Wentao, Daning Li, Jiaoyang Du, Xiangyu Gao, Wen Gu, Fanfan Zhao, Xiaojie Feng, and Hong Yan. "An Intelligent Diagnosis Method of Brain MRI Tumor Segmentation Using Deep Convolutional Neural Network and SVM Algorithm." Computational and Mathematical Methods in Medicine 2020.

[58] Hoseini, Farnaz, Asadollah Shahbahrami, and Peyman Bayat. "An efficient implementation of deep convolutional neural networks for MRI segmentation." Journal of digital imaging 31, no. 5 (2018): 738-747. 
[59] Baid, Ujjwal, Sanjay Talbar, Swapnil Rane, Sudeep Gupta, Meenakshi H. Thakur, Aliasgar Moiyadi, Nilesh Sable, Mayuresh Akolkar, and Abhishek Mahajan. "A Novel Approach for Fully Automatic Intra-Tumor Segmentation With 3D U-Net Architecture for Gliomas." Frontiers in Computational Neuroscience 14 (2020).

[60] Tjahyaningtijas, H. Peni Agustin. "Brain tumor image segmentation in MRI image." In Proc. IOP Conf. Ser., Mater. Sci. Eng., vol. 336, 2018.

[61] Cui, Shaoguo, Lei Mao, Jingfeng Jiang, Chang Liu, and Shuyu Xiong. "Automatic semantic segmentation of brain gliomas from MRI images using a deep cascaded neural network." Journal of healthcare engineering 2018.

[62] Sourav Singh, Manali Bhavsar , Rasika Mahadeshwar, Sumeet Rathod, Mrs. Disha Wankhede "Predicting Idh1 Mutation And 1p19q Co-Deletion Status For Brain Tumor", International Journal of Advanced Science and Technology Vol. 29, No. 4s, (2020), pp.1196-1204

[63] https://brainweb.bic.mni.mcgill.ca/

[64] https://www.oasis-brains.org/ 